\title{
Anti-Inflammatory Activity of Four Triterpenoids Isolated from Poriae Cutis
}

\author{
Lijia Zhang ${ }^{1}$, Mengzhou Yin ${ }^{1}$, Xi Feng ${ }^{2}$, Salam A. Ibrahim ${ }^{3} \mathbb{D}$, Ying Liu ${ }^{1}$ (D) and Wen Huang ${ }^{1, * \mathbb{D}}$ \\ 1 College of Food Science and Technology, Huazhong Agricultural University, Wuhan 430070, China; \\ 13297021290@163.com (L.Z.); mz324666@163.com (M.Y.); yingliu@mail.hzau.edu.cn (Y.L.) \\ 2 Department of Nutrition, Food Science and Packaging, San Jose State University, San Jose, CA 95192, USA; \\ xi.feng@sjsu.edu \\ 3 Department of Family and Consumer Sciences, North Carolina Agricultural and Technical State University, \\ 171 Carver Hall, Greensboro, NC 27411, USA; ibrah001@ncat.edu \\ * Correspondence: huangwen@mail.hzau.edu.cn; Tel.: +86-136-5980-7072
}

Citation: Zhang, L.; Yin, M.; Feng, X.; Ibrahim, S.A.; Liu, Y.; Huang, W. Anti-Inflammatory Activity of Four Triterpenoids Isolated from Poriae Cutis. Foods 2021, 10, 3155. https:// doi.org/10.3390/foods10123155

Academic Editor: Laura Jaime

Received: 18 November 2021 Accepted: 15 December 2021 Published: 20 December 2021

Publisher's Note: MDPI stays neutral with regard to jurisdictional claims in published maps and institutional affiliations.

Copyright: (c) 2021 by the authors Licensee MDPI, Basel, Switzerland. This article is an open access article distributed under the terms and conditions of the Creative Commons Attribution (CC BY) license (https:// creativecommons.org/licenses/by/ $4.0 /)$

\begin{abstract}
In this study, triterpenoid compounds from Poriae Cutis were separated by high-speed countercurrent chromatography (HSCCC) and identified using ultra-high performance liquid chromatography quadrupole time-of-flight tandem mass spectrometry (UHPLC-QTOF-MS/MS) and nuclear magnetic resonance (NMR). The in vitro anti-inflammatory activities of the purified triterpenoids on RAW 264.7 cells were also investigated. Triterpenoids, poricoic acid B, poricoic acid A, dehydrotrametenolic acid, and dehydroeburicoic acid were obtained; their levels of purity were $90 \%, 92 \%$, $93 \%$, and $96 \%$, respectively. The results indicated that poricoic acid B had higher anti-inflammatory activity than those of poricoic acid A by inhibiting the generation of NO in lipopolysaccharide (LPS)-induced RAW 264.7 cells. However, dehydrotrametenolic acid and dehydroeburicoic acid had no anti-inflammatory activity. In addition, the production of cytokines (TNF- $\alpha$, IL-1 $\beta$, and IL-6) in cells treated with poricoic acid B decreased in a dose-dependent manner in the concentration range from 10 to $40 \mu \mathrm{g} / \mathrm{mL}$. The results provide evidence for the use of Poriae Cutis as a natural anti-inflammatory agent in medicines and functional foods.
\end{abstract}

Keywords: Poriae Cutis; triterpenoid; purification; identification; anti-inflammatory

\section{Introduction}

Poria cocos (Schw.) Wolf ("fuling" in Chinese) is a widely used medicinal fungus. According to the Chinese Pharmacopoeia, about one-tenth of traditional Chinese medicine preparations include $P$. cocos [1]. Additionally, $P$. cocos is used as a dietary supplement and in foods such as soups, dishes, snacks, and desserts for its health-promoting benefits. It can be divided into four parts, namely, Poriae Cutis (the epidermis, "fulingpi" in Chinese), Rubra Poria (the pink part near the epidermis), White Poria (the middle part, "baifuling" in Chinese), and Poria cum Radix Pini (the middle-plus-inner part, "fushen" in Chinese) [2]. Different parts of Poria cocos have different pharmacological effects and clinical applications. Both P. cocos and Poriae Cutis are officially included in the Chinese Pharmacopoeia. Clearing damp and promoting diuresis are traditional Chinese medicinal uses for Poria cocos. Poriae Cutis is mainly used to promote urination in clinics. Nowadays, White Poria is widely used due to its diuretic, tonic, and sedative functions. However, Poriae Cutis is treated as agricultural waste during the harvest periods and is rarely used $[1,3]$.

The functional constituents in $P$. cocos mainly contain triterpenes and polysaccharides, while the main bioactive constituent of Poriae Cutis is triterpenoid. Triterpenes isolated from $P$. cocos can be divided into four subgroups based on their chemical structure: lanosta-8-ene type, lanosta-7,9(11)-diene type, 3,4-seco-lanostan-8-ene type, and 3,4-seco-lanostan-7,9(11)diene type compounds [4]. In prior research, 59 types of triterpenoids were separated from $P$. cocos; 41 types were isolated from Poriae Cutis $[2,4]$. The total content of triterpenes 
and bioactive triterpenes in Poriae Cutis was found to be higher than in the sclerotia of $P$. $\operatorname{cocos}[1,5-7]$, and triterpenoids from the sclerotia of $P$. cocos showed anti-inflammatory activity [8,9]. It was reported that seco-lanostane triterpenoid come from the sclerotia of $P$. cocos and could be a promising lead compound for the development of anti-inflammatory agents for use in pharmaceuticals and functional foods [8]. Triterpenes have attracted attention due to their anti-inflammatory [8,10,11], anticancer [12-14], and diuretic properties $[3,15]$. Of the many reports on the biological activity of triterpenes in Poriae Cutis, most have focused on antitumor and diuretic activities. However, there are few studies on the anti-inflammatory activity of triterpenoids in Poriae Cutis.

The objectives of this study were to separate triterpenoids from Poriae Cutis by HSCCC chromatography, and then identify them with UHPLC-QTOF-MS/MS and NMR spectra. The anti-inflammatory activity of triterpenoids was also determined using RAW 264.7 mouse macrophage cells.

\section{Materials and Methods}

\subsection{Crude Sample Preparation}

Poria cocos was obtained from the local market in Lou tian (Hubei Province, China). The powder of Poriae Cutis (50 g) was extracted with n-butanol-water $(1: 1, v / v)$ solution $(2 \mathrm{~L})$ at $60{ }^{\circ} \mathrm{C}$ under reflux for $40 \mathrm{~min}$. After filtration, the filtrate was concentrated by a rotary evaporator. Concentrated filtrate was suspended in distilled water and extracted with ethyl acetate three times. The combined ethyl acetate solution was evaporated to dryness to yield crude triterpenes from Poriae Cutis, which were used for subsequent HSCCC separation [16].

\subsection{HSCCC Separation}

A high-speed counter-current chromatography (HSCCC) with a two-phase solvent system (TBE-300C, Shanghai Tauto Biotech Co., Ltd., Shanghai, China) with n-hexaneethyl acetate-MeOH-water $(3: 6: 4: 2, v / v / v / v)$ was used; the stationary phase was the upper phase, and the mobile phase was the lower phase. Crude triterpene $(100 \mathrm{mg})$ was dissolved in the mobile phase $(10 \mathrm{~mL})$. After two-phase ultrasonic degassed for $15 \mathrm{~min}$, the sample solution was injected through the injection valve when it reached hydrodynamic equilibrium. Following the stationary phase, the column was filled entirely at a flow rate of $30 \mathrm{~mL} / \mathrm{min}$; the lower phase was pumped at a flow rate of $3 \mathrm{~mL} / \mathrm{min}$. The rotation was set at $800 \mathrm{rpm}$ with a detection wavelength of $242 \mathrm{~nm}$. Six peaks (P-1, P-2, P-3, P-4, P-5, and P-6) were separately collected and evaporated to dryness to yield purified fractions [16].

\subsection{HPLC Analysis}

The six purified fractions (P-1, P-2, P-3, P-4, P-5, and P-6) were dissolved in methanol and then filtered by a $0.45 \mu \mathrm{m}$ syringe filter for HPLC analysis. HPLC (e2695, Waters Corporation, Milford, MA, USA) analyses were performed with an InertSustainTMAQ-C18 column ( $4.6 \mathrm{~mm} \times 250 \mathrm{~mm}, 5 \mu \mathrm{m}$, Shimadzu, Shanghai, China) at $25^{\circ} \mathrm{C}$. The mobile phase was composed of solvent A $(0.05 \%(v / v)$ phosphoric acid) and solvent B (methanol). The gradient mode was as follows: 0-15 min, 30-25\% A, 70-75\% B; 15-25 min, 25-21\% A, $75-79 \%$ B; 25-40 $\mathrm{min}, 21-10 \% \mathrm{~A}, 79-90 \% \mathrm{~B} ; 40-65 \mathrm{~min}, 10 \% \mathrm{~A}, 90 \% \mathrm{~B}$. The injection volume was $10 \mu \mathrm{L}$ and the flow rate was $1.0 \mathrm{~mL} / \mathrm{min}$ with a detection wavelength of $242 \mathrm{~nm}$.

\subsection{UHPLC-QTOF-MS/MS Analysis}

UHPLC (1100-VL, Waters Corporation, Milford, MA, USA) analyses were performed with an ACQUITY UPLC ${ }^{\circledR}$ BEH C18 column $(2.1 \mathrm{~mm} \times 100 \mathrm{~mm}, 1.7 \mu \mathrm{m}$, Waters Corporation, Milford, MA, USA) at $30{ }^{\circ} \mathrm{C}$. The mobile phase was composed of solvent A (water) and solvent $\mathrm{B}$ (methanol). The gradient mode was as follows: 0-7 min, 44-23\% A, 56-77\% B; 7-14 min, 23-15\% A, 77-85\% B; 14-24 min, 15\% A, 85\% B. The injection volume was $1 \mu \mathrm{L}$ and the flow rate was kept at $0.3 \mathrm{~mL} / \mathrm{min}$. Mass spectrometry conditions were set with the ESI source in negative ionization mode, drying gas temperature at $350{ }^{\circ} \mathrm{C}$, drying gas flow 
$600 \mathrm{~L} / \mathrm{h}$, and capillary voltage $2.4 \mathrm{kV}$. The full scan range of $\mathrm{m} / \mathrm{z} 10-1000$ was performed for mass detection.

\subsection{NMR Spectra Analysis}

Samples were dissolved in methanol-d4 and pyridine-d5 for NMR spectra measurement. NMR spectra analyses were performed by an AVANCE III $400 \mathrm{MHz}$ NMR spectrometer (Bruker Corporation, Zurich, Switzerland). ${ }^{1} \mathrm{H}-\mathrm{NMR}$ and ${ }^{13} \mathrm{C}-\mathrm{NMR}$ data were analyzed and processed by Meatrenove 14.0.

\subsection{Anti-Inflammatory Activity of Triterpenoids from Poriae Cutis In Vitro 2.6.1. Cell Viability Assay}

RAW 264.7 cells (acquired from Chinese Academy of Sciences) were maintained in Dulbecco's modified Eagle's medium (DMEM, Gibco Company, New York, NY, USA), supplemented with fetal bovine serum (FBS) $(10 \%, v / v)$ (Gibco Company, USA) and antibiotics $(1 \%, v / v)$ (penicillin $(100 \mathrm{U} / \mathrm{mL})$ and streptomycin $(100 \mu \mathrm{g} / \mathrm{mL}))$ in a humidified incubator at $37{ }^{\circ} \mathrm{C}$ with $5 \% \mathrm{CO}_{2}$. Cells were plated into 96 -well plates at $1 \times 10^{5}$ cells $/ \mathrm{mL}$. After incubating for $24 \mathrm{~h}$, the RAW 264.7 cells were added to $100 \mu \mathrm{L}$ of four triterpenoids from Poriae Cutis with different concentrations (poricoic acid A and poricoic acid B: 10, 20, 30, 40, and $50 \mu \mathrm{g} / \mathrm{mL}$; dehydrotrametenolic acid and dehydroeburicoic acid: $0.125,0.25,0.5,1$, and $5 \mu \mathrm{g} / \mathrm{mL}$ ), DMEM basic medium (control group) or lipopolysaccharide (LPS) (Sigma Company, St. Louis, MO, USA) $(1 \mu \mathrm{g} / \mathrm{mL}$, positive control) for $24 \mathrm{~h}$. Next, the growth medium was removed and $100 \mu \mathrm{L}$ of 3-(4,5-dimethylthiazole-2)-2,5-diphenyltetrazolium bromine salt (MTT) (American Biosharp Company, Houston, TX, USA) solution $(0.5 \mathrm{mg} / \mathrm{mL})$ was added for $4 \mathrm{~h}$. Subsequently, the conditioned medium was eliminated and DMSO (150 $\mu \mathrm{L})$ was added, and cell viability was detected using optical density (OD) at $490 \mathrm{~nm}$ using a Multiskan GO automatic microplate reader (Thermo, Waltham, MA, USA) [17].

\subsubsection{NO Determination}

RAW 264.7 cells were plated into 24 -well plates $\left(8 \times 10^{5}\right.$ cells $\left./ \mathrm{mL}\right)$ and cultured as described above. The cells were then simultaneously induced with LPS $(1 \mu \mathrm{g} / \mathrm{mL})$ and various doses of the four triterpenoids from Poriae Cutis (poricoic acid A and poricoic acid B, 10, 20, 30, and $40 \mu \mathrm{g} / \mathrm{mL}$; dehydrotrametenolic acid and dehydroeburicoic acid, $0.125,0.25,0.5$, and $1 \mu \mathrm{g} / \mathrm{mL}$ ), DMEM basic medium (control group), or LPS $(1 \mu \mathrm{g} / \mathrm{mL}$, positive control) for $24 \mathrm{~h}$. Next, the conditioned medium $(100 \mu \mathrm{L})$ was mixed with Griess reagent $(100 \mu \mathrm{L})$ in each well and incubated for $10 \mathrm{~min}$. Finally, the absorbance at $540 \mathrm{~nm}$ was measured using a Multiskan GO automatic microplate reader. $\mathrm{NaNO}_{2}$ was used as a standard to calculate the released NO concentration [18].

\subsubsection{Cytokines Assays of TNF- $\alpha$, IL- $1 \beta$, and IL-6}

RAW 264.7 cells were cultured in 24-well plates $\left(8 \times 10^{5}\right.$ cells $\left./ \mathrm{mL}\right)$, to which LPS $(1 \mu \mathrm{g} / \mathrm{mL})$ was added and various doses of poricoic acid B (10, 20 and $40 \mu \mathrm{g} / \mathrm{mL})$, DMEM basic medium (control group), or LPS $(1 \mu \mathrm{g} / \mathrm{mL}$, positive control) for $24 \mathrm{~h}$. The cells were then centrifuge at $2400 \mathrm{r} / \mathrm{min}$ for $5 \mathrm{~min}$ and the supernatant was collected. The secretions of cytokines in the supernatant were tested using a TNF- $\alpha$, IL-1 $\beta$, and IL-6 ELISA kit (Guangzhou Xinbosheng Biotechnology Co., Ltd., Guangzhou, China).

\subsection{Data Analysis}

The structural formula of the compound was drawn by ChemDraw 16.0. Statistical differences between treatments were determined using ANOVA and Duncan's or Tamhane's test at a significance level of 0.05 or 0.01 . 


\section{Results and Discussion}

\subsection{HSCCC Separation}

HSCCC is widely utilized in the isolating of natural products. It has high stability, good reproducibility, the non-requirement of a solid carrier, a low risk of nonreversible adsorption, and a high sample recovery rate $[19,20]$. Fractions of $P$. cocos were often obtained by means of a silica gel column chromatography and eluted successively [21], but this requires a complicated operation process and causes sample loss. It was reported that HSCCC is an effective methodology for isolating of high-purity triterpenoid compounds in Inonotus obliquus [20]. In the present study, HSCCC was used to separate triterpenoid compounds from Poriae Cutis. As shown in Figure 1, compounds P-1 (14.37\%), P-2 (7.93\%), P-3 (20.38\%), P-4 (35.64\%), P-5 (19.07\%), and P-6 (2.59\%) were efficiently separated within $360 \mathrm{~min}$ with a $50 \%$ retention rate. After elution, all of the fractions were dried and the collected fractions were identified by HPLC.

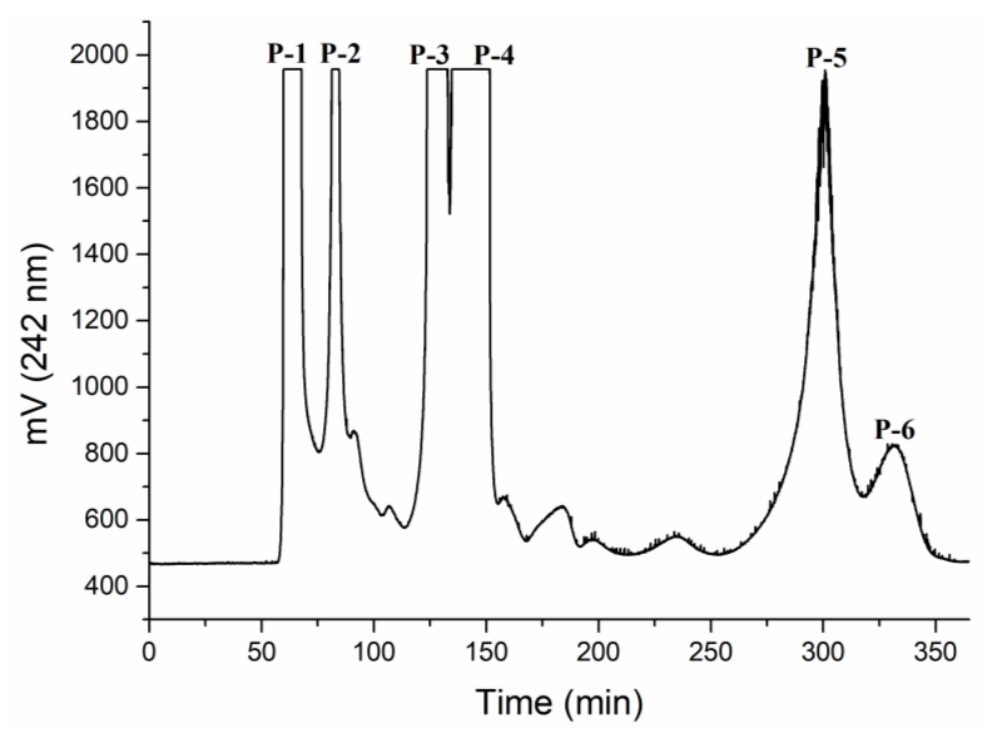

Figure 1. Elution profile of triterpenoids by HSCCC chromatogram. n-hexane-ethyl acetate-MeOHwater (3:6:4:2, $v / v / v / v)$; flow rate: $3 \mathrm{~mL} / \mathrm{min}$; detection wavelength: $242 \mathrm{~nm}$; rotational speed: 800 rpm; sample size: $100 \mathrm{mg}$.

\subsection{HPLC Analysis}

Among the six fractions collected through HSCCC, only P-3, P-4, P-5, and P-6 had a purity of higher than $90 \%(90 \%, 92 \%, 93 \%$, and $96 \%$, respectively). Therefore, the follow-up experiments focused on these four fractions, and we named them compound-1, compound$\mathbf{2}$, compound-3, and compound-4, respectively. A total of $5.20 \mathrm{mg}$ of compound-1, $20.50 \mathrm{mg}$ of compound-2, $0.4 \mathrm{mg}$ of compound-3, and $0.1 \mathrm{mg}$ of compound-4 were separated from the above sample ( $100 \mathrm{mg}$ ) by HSCCC. As shown in Figure 2, the retention time of the four fractions was $33.23 \mathrm{~min}$ (compound-1), $36.20 \mathrm{~min}$ (compound-2), $55.45 \mathrm{~min}$ (compound-3), and $59.88 \mathrm{~min}$ (compound-4). 


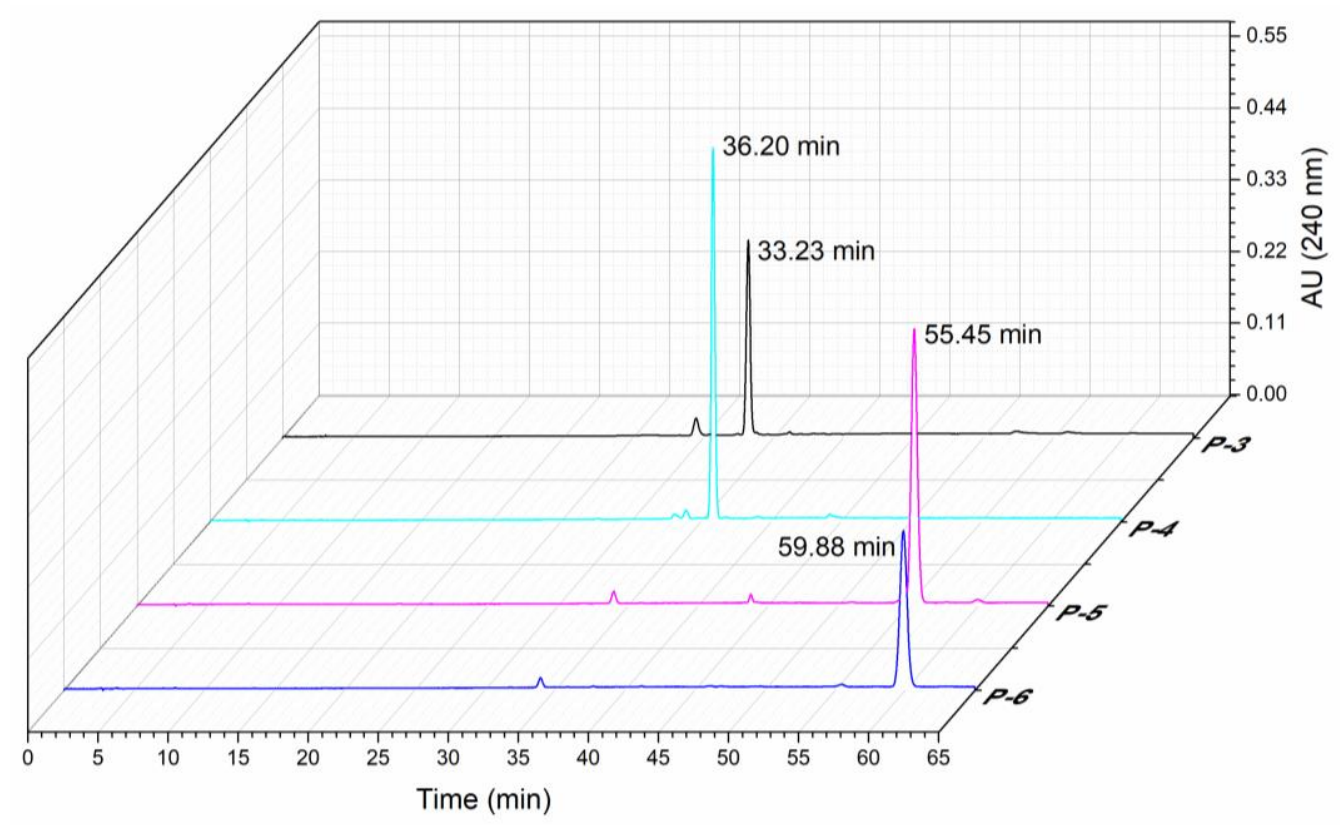

Figure 2. HPLC chromatogram of four kinds of triterpenoids obtained by HSCCC.

\subsection{Identification of Compounds}

The structures of all compounds were characterized by ESI-MS, ${ }^{1} \mathrm{H}-\mathrm{NMR}$, and ${ }^{13} \mathrm{C}-$ NMR spectra and are shown in Figures S1-S4. Compound-1 (Figure S1): ESI-MS $(m / z)$ : $483.3104[\mathrm{M}-\mathrm{H}]^{-}$. UV $\lambda_{\max }: 242 \mathrm{~nm}$. The ${ }^{1} \mathrm{H}-$ and ${ }^{13} \mathrm{C}-\mathrm{NMR}$ spectra revealed $\mathrm{H}-11(\delta 5.21) / \mathrm{C}-$

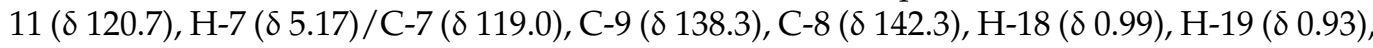

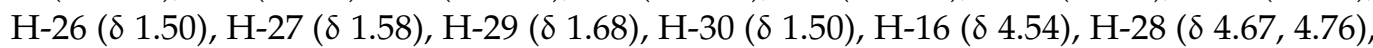
H-24 ( $\delta 5.27), C-3(\delta 178.3), C-21(\delta 180.0)$. Based on the structural features of triterpene compounds and the data given in the literature, compound-1 was identified as poricoic acid B [1,17,20,22-24]. Poricoic acid B belongs to the 3,4-seco-lanostan-7,9(11)-diene type triterpenes [4]. Its chemical structure is shown in Figure 3.

Compound-2 (Figure S2): ESI-MS $(m / z): 497.3266$ [M-H]'. UV $\lambda_{\max }: 242 \mathrm{~nm}$. The ${ }^{1} \mathrm{H}-$ and ${ }^{13} \mathrm{C}-\mathrm{NMR}$ spectra presented H-11 ( $\delta$ 5.27)/C-11 ( $\delta$ 119.5), H-7 $(\delta$ 5.21)/C-7 $(\delta$

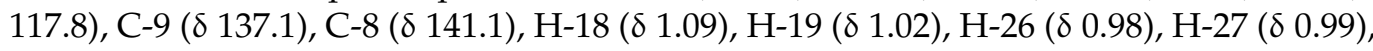

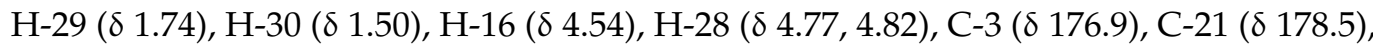
C-24 ( $\delta 155.3), C-25$ ( $\delta 33.6), C-31$ ( $\delta 106.7)$. Based on the structural features of triterpene compounds and the data given in the literature, compound- 4 was identified as poricoic acid A $[1,16,19,21,22]$. Poricoic acid A belongs to the 3,4-seco-lanostan-7,9(1 1)-diene type triterpenes [4]. Its chemical structure is shown in Figure 3.

Compound-3 (Figure S3): ESI-MS $(m / z): 453.3370[\mathrm{M}-\mathrm{H}]^{-}$. UV $\lambda_{\max }: 243 \mathrm{~nm}$. The ${ }^{1} \mathrm{H}-$ and ${ }^{13} \mathrm{C}-\mathrm{NMR}$ spectra indicated that $\mathrm{H}-11$ ( $\delta$ 5.37)/C-11 ( $\left.\delta 116.5\right), \mathrm{H}-7$ ( $\delta$ 5.61)/C-7

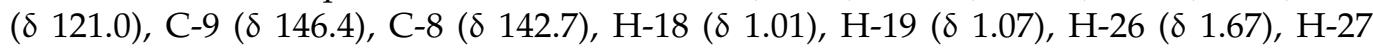

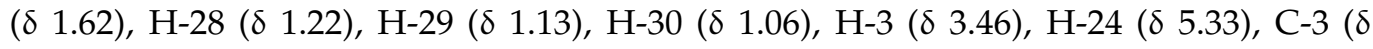
77.9), C-21 ( $\delta 178.9), C-24$ ( $\delta 124.8), C-25$ ( $\delta 131.5)$. Based on the structural features of triterpene compounds and the data given in the literature, compound- 4 was identified as dehydrotrametenolic acid [1,2,19]. Dehydrotrametenolic acid belongs to the lanosta-7,9(11)diene type triterpenes [4]. Its chemical structure is shown in Figure 3.

Compound-4 (Figure S4): ESI-MS ( $\mathrm{m} / \mathrm{z}): 467.3518$ [M-H]-. UV $\lambda_{\max }: 243 \mathrm{~nm}$. The ${ }^{1} \mathrm{H}-$

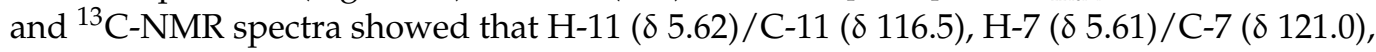

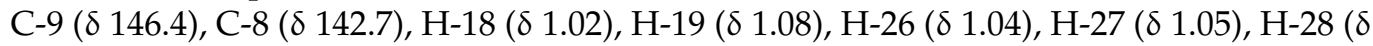

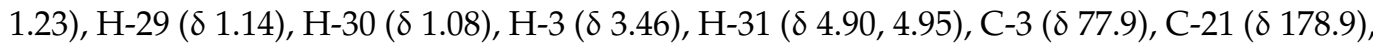
C-24 ( $\delta 160.8), C-31(\delta 106.8)$. Based on the structural features of triterpene compounds and the data given in the literature, compound- $\mathbf{4}$ was identified as dehydroeburicoic acid [1,24]. 
Dehydroeburicoic acid belongs to the lanosta-7,9(11)-diene type triterpenes [4]. Its chemical structure is shown in Figure 3.

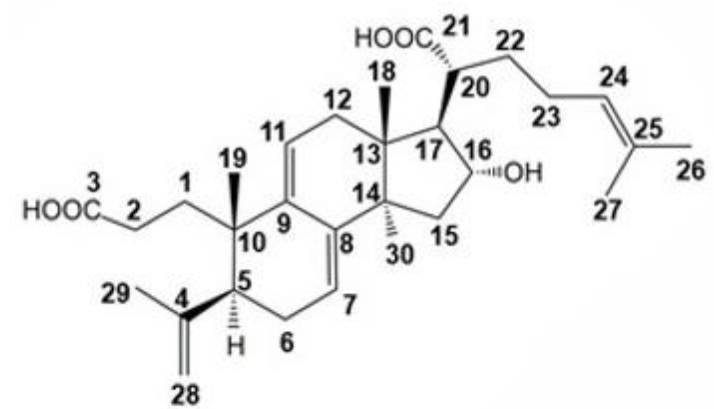

Compound-1 (P-3) Poricoic acid B

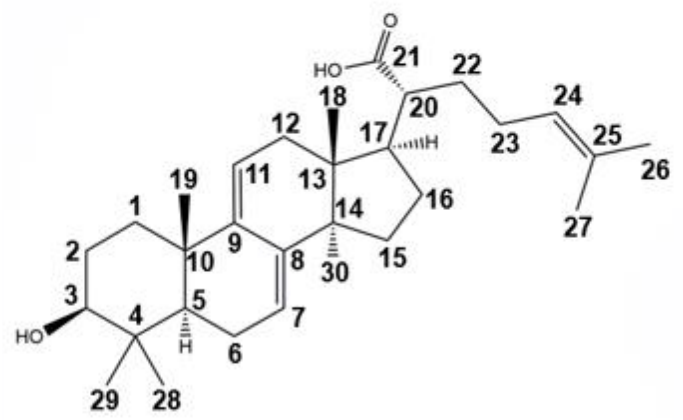

Compound-3 (P-5) Dehydrotrametenolic acid

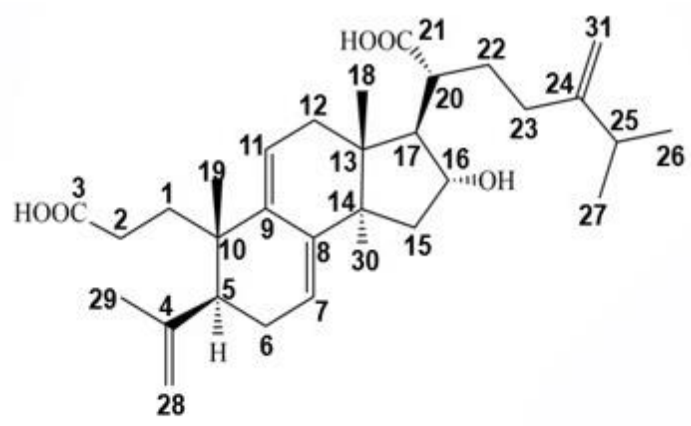

Compound-2 (P-4) Poricoic acid A

Figure 3. The structures of compounds 1-4.

3.4. In Vitro Anti-Inflammatory Activity of Triterpenoids

\subsubsection{Effects of Triterpenoids on Viability of RAW264.7 Cells}

The cytotoxic effect of poricoic acid A, poricoic acid B, dehydrotrametenolic acid, and dehydroeburicoic acid on RAW 264.7 cells was evaluated by an MTT assay. It is generally acknowledged that when adding samples, if the cell viability is below $90 \%$, it indicates cytotoxicity. If the cell viability is higher than this critical value, the sample is non-cytotoxic and can be used for further research [25]. The viabilities of the RAW 264.7 cells of the four triterpenoids from Poriae Cutis were different and are shown in Figure 4. As shown in Figure $4 \mathrm{~A}$, the two treatment groups of poricoic acid A and poricoic acid B at concentrations of $10-30 \mu \mathrm{g} / \mathrm{mL}$ and $10-40 \mu \mathrm{g} / \mathrm{mL}$ had no cytotoxicity on macrophages. As shown in Figure 4B, the non-cytotoxic concentrations of dehydrotrametenolic acid and dehydroeburicoic acid were $0.125-1$ and $0.125-0.5 \mu \mathrm{g} / \mathrm{mL}$, respectively. However, Lee et al. [8] reported a the cell viability of $>90 \%$ for $n$-butanol fraction concentrations of up to $50 \mu \mathrm{g} / \mathrm{mL}$. This could be due to the differences in the sample purities. 

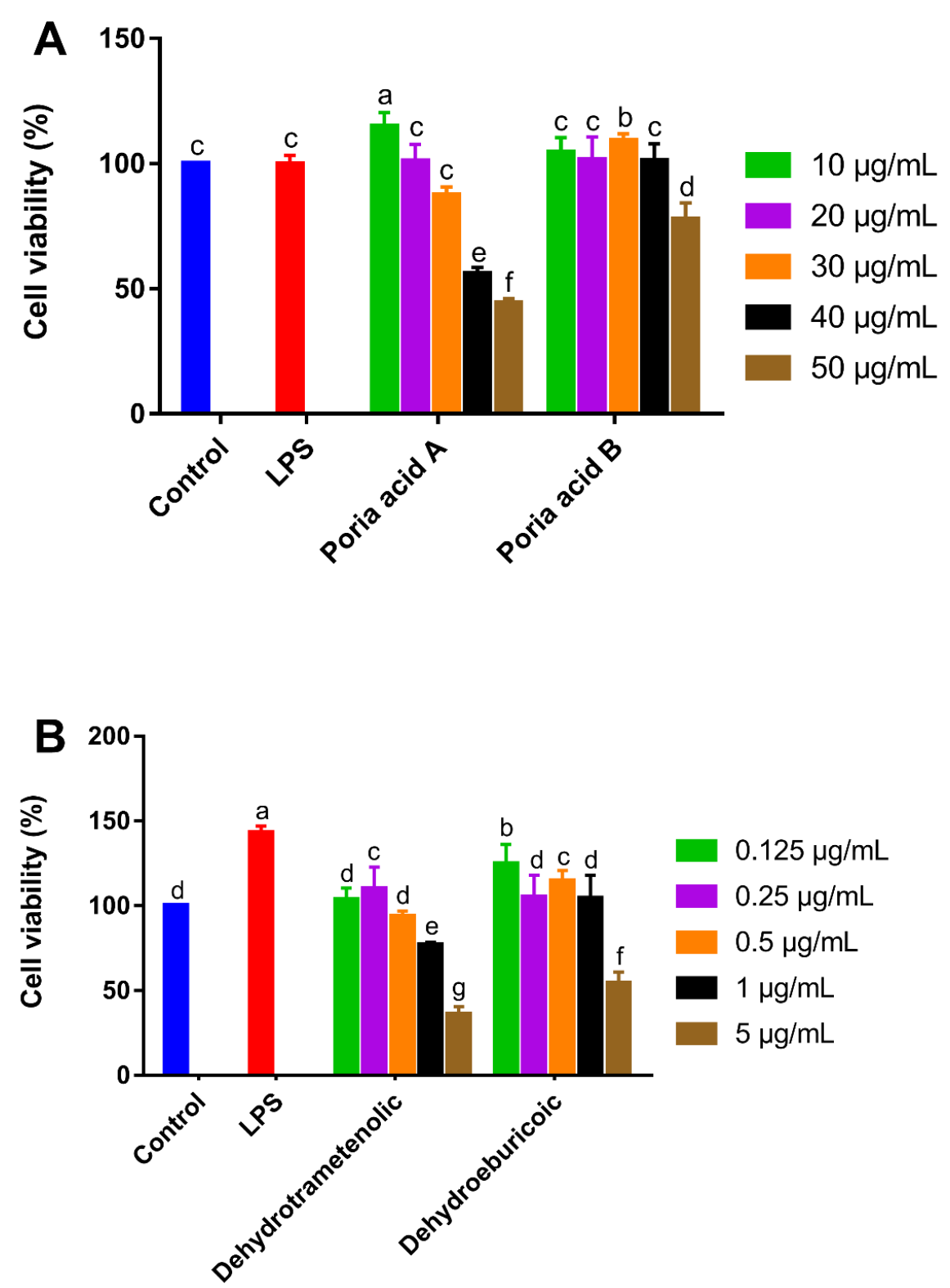

Figure 4. The effects of various concentrations of poricoic acid A and poricoic acid B (A), dehydrotrametenolic acid and dehydroeburicoic acid (B) on the cell viabilities of murine macrophage cells (RAW 264.7). The values are expressed as the mean $\pm \mathrm{SD}(n=3)$. Different letters (a-g) indicate significant differences $(p<0.05)$. LPS, lipopolysaccharide.

In summary, the maximum concentrations of poricoic acid A, poricoic acid B, dehydrotrametenolic acid, and dehydroeburicoic acid without significant cell activity inhibition on RAW264.7 cells were $30,40,1$, and $0.5 \mu \mathrm{g} / \mathrm{mL}$, respectively. The difference may be related to their chemical structures. Poricoic acid A and poricoic acid B belong to 3,4seco-lanostan-7,9(11)-diene type triterpenes, which were less cytotoxic to RAW264.7 cells; dehydrotrametenolic acid and dehydroeburicoic acid are both lanosta-7,9(11)-diene type triterpenes, which were reported to have a higher cytotoxicity [4]. Dong et al. [26] also reported that seco-lanostane triterpenoid from the epidermis of Poria cocos showed weak cytotoxic activities on MGC-803 and HepG2 cell lines.

\subsubsection{Effects of Triterpenoids on NO Production of RAW 264.7 Cells}

Nitric oxide (NO) has been acknowledged as an important signaling molecule related to inflammatory responses [24]. Natural compounds were reported to affect the secretion 
of NO, which helped to develop drugs to treat inflammatory diseases $[27,28]$. LPS is an important proinflammatory factors, which can increase the production of NO [29]. The inhibitory effects of four triterpenoids from Poriae Cutis on NO production in LPS-induced RAW 264.7 cells in co-culture experiments are shown in Figure 5. The NO production level of macrophages in the control group was weak, but the NO production level increased significantly after LPS stimulation, which indicated that RAW264.7 cells had already developed an inflammatory state under the induction of LPS. As shown in Figure 5A, the RAW 264.7 cells treated with either poricoic acid A at concentrations of $10-20 \mu \mathrm{g} / \mathrm{mL}$ or poricoic acid B at concentrations of $10 \mu \mathrm{g} / \mathrm{mL}$ showed no change in the secretions of $\mathrm{NO}(p>0.05)$, but the change became significant with a further increase in concentration. The NO production in cells treated with poricoic acid A significantly decreased to $47 \%$ at $30 \mu \mathrm{g} / \mathrm{mL}$. Poricoic acid B dose-dependently decreased NO production at concentrations of 20,30 , and $40 \mu \mathrm{g} / \mathrm{mL}$, and considerably suppressed NO production to $29 \%, 34 \%$, and $68 \%$, respectively. However, we found that the $\mathrm{IC}_{50}$ of poricoic acid $\mathrm{A}$ that inhibited NO production in LPS-activated cells was $18.12 \mu \mathrm{M}(8.77 \mu \mathrm{g} / \mathrm{mL})$ [8]. As shown in Figure 5B, dehydrotrametenolic acid and dehydroeburicoic acid had no effect on the secretions of NO and they seemed to have no anti-inflammatory activity. The results are consistent with those in a previous report [9]. Additionally consistent with the report is that the inhibition of NO of seco-lanostane triterpenoid was higher than that of lanostane triterpenoid [8].
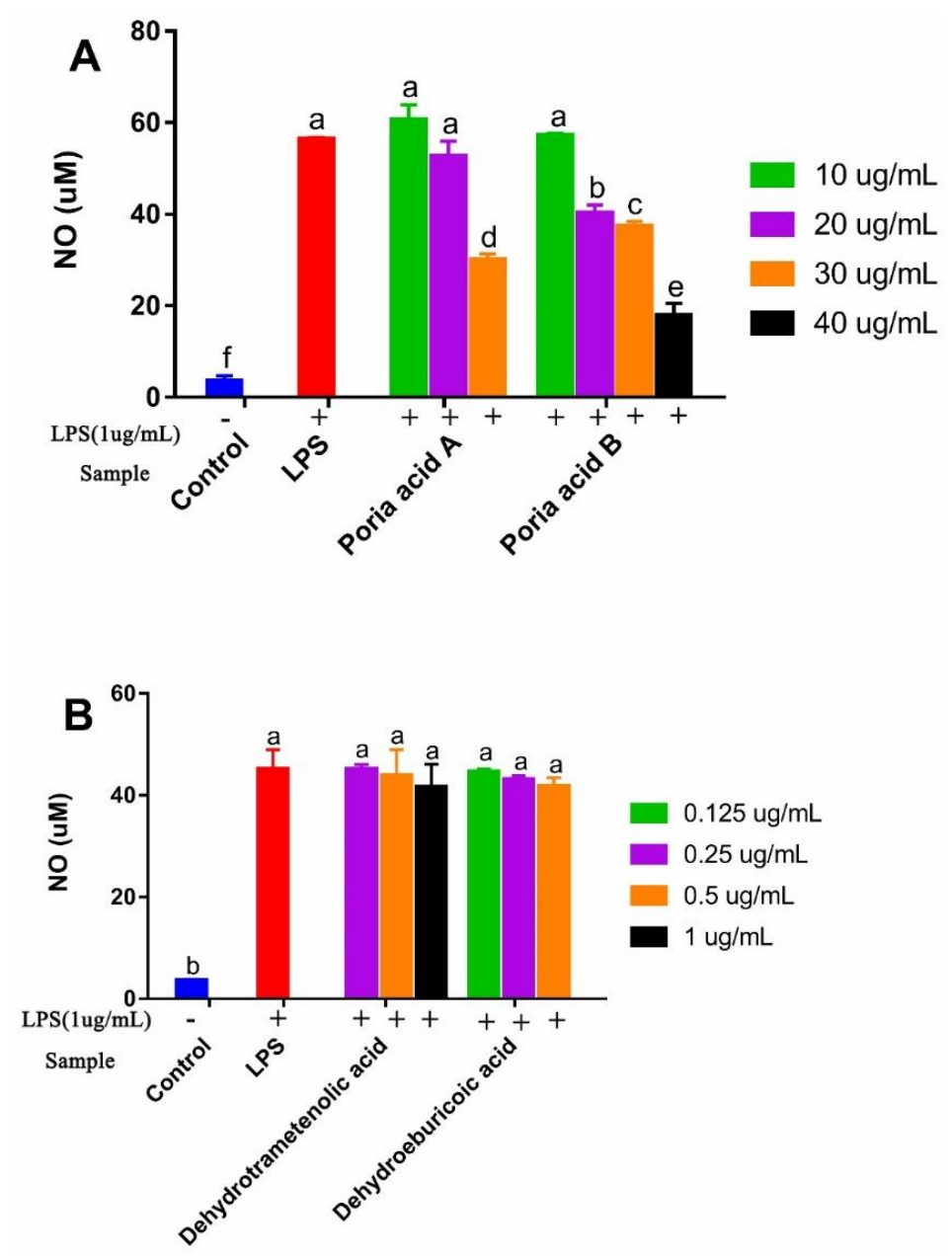

Figure 5. The effects of various concentrations of poricoic acid A and poricoic acid B (A), dehydrotrametenolic acid and dehydroeburicoic acid (B) on the NO production in lipopolysaccharide (LPS)-stimulated murine macrophage cells (RAW 264.7). The values are presented as the mean $\pm \mathrm{SD}$ $(n=3)$. Different letters $(\mathrm{a}-\mathrm{f})$ indicate significant differences $(p<0.05)$. 


\subsubsection{Effects of Poricoic Acid B on Cytokines Production of RAW 264.7 Cells}

The anti-inflammatory activities of four triterpenes (poricoic acid A, poricoic acid B, dehydrotrametenolic acid, and dehydroeburicoic acid) were assessed by an LPS-stimulated NO production model on RAW 264.7 cells. The results revealed that poricoic acid B exhibited a strong NO production inhibitory activity in a dose-dependent manner. Thus, the secretion of cytokines including tumor necrosis factor (TNF)- $\alpha$, interleukin (IL)- $1 \beta$, and interleukin (IL)-6 was measured with poricoic acid B in a medium of RAW 264.7 in co-culture experiments, which were quantified by an ELISA assay.

It has been reported that LPS-induced RAW 264.7 cells secrete pro-inflammatory factors TNF- $\alpha$, IL-1 $\beta$, and IL- 6 during the inflammation process [30,31]. As shown in Figure 6 , the production of TNF- $\alpha$, IL- $1 \beta$, and IL- 6 by cells treated with poricoic acid B showed a dose-dependent decrease in the concentration range from 10 to $40 \mu \mathrm{g} / \mathrm{mL}$ in the co-culture experiments, all of which were more than in the control group $(193 \mathrm{pg} / \mathrm{mL})$, but less than in the LPS-treated group $\left(2.63 \times 10^{5} \mathrm{pg} / \mathrm{mL}\right)$. The result indicated that LPS increased the release of pro-inflammatory cytokines [28], and poricoic acid $B$ reduced the secretion of cytokines. TNF- $\alpha$ secretion from the poricoic acid B treated group decreased significantly in the concentration range of $10-40 \mu \mathrm{g} / \mathrm{mL}$. The value was the lowest at a concentration of $20 \mu \mathrm{g} / \mathrm{mL}$, which was reduced by $32 \%$ compared to that in the LPS-treated group (Figure 6A). The poricoic acid B treated group did not show a significant difference in IL-1 $\beta$ at relatively lower concentrations $(10 \mu \mathrm{g} / \mathrm{mL})$ but displayed significant decreases at higher concentrations. At the doses of 20 and $40 \mu \mathrm{g} / \mathrm{mL}$, the secretion of IL-1 $\beta$ decreased by $41 \%$ and $77 \%$ in comparison to the LPS-treated group (Figure $6 \mathrm{~B}$ ), respectively. With the increase in the concentration of poricoic acid B, the amount of IL-6 production gradually decreased. Compared to the LPS-treated group, the secretion of IL- 6 decreased by $30 \%$, $53 \%$, and $66 \%$ under the poricoic acid B treatment concentrations of 10,20 , and $40 \mu \mathrm{g} / \mathrm{mL}$, respectively (Figure 6C). 3,4-seco-dammarane triterpenoid saponins isolated from the leaves of Cyclocarya paliurus showed similar inhibition of IL-6 and TNF- $\alpha$ [32].

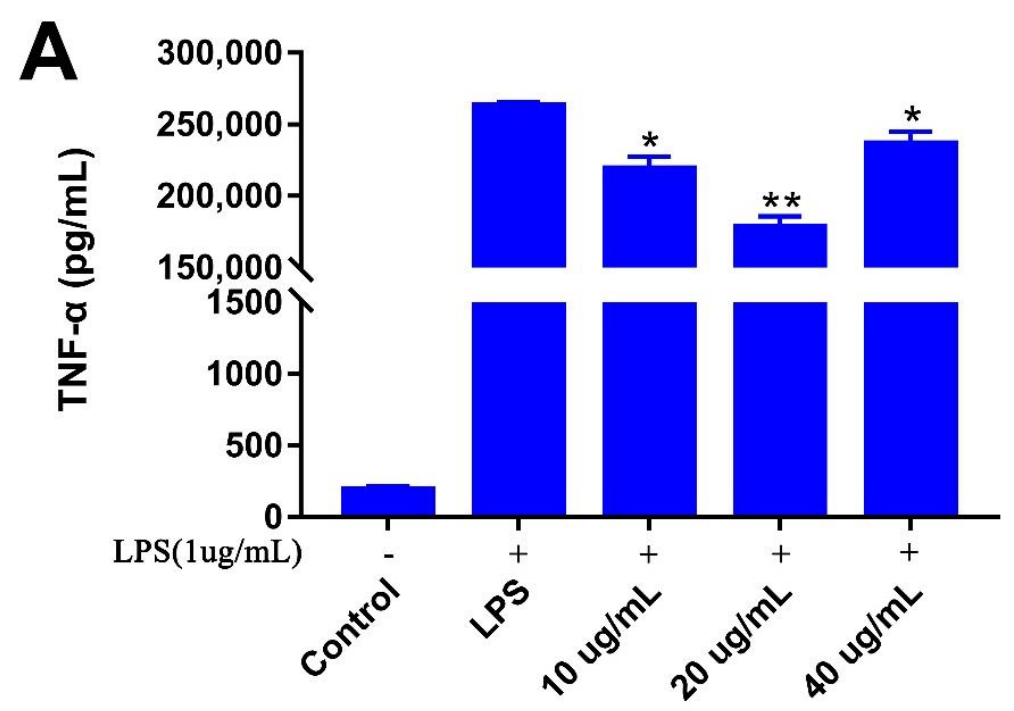

Figure 6. Cont. 

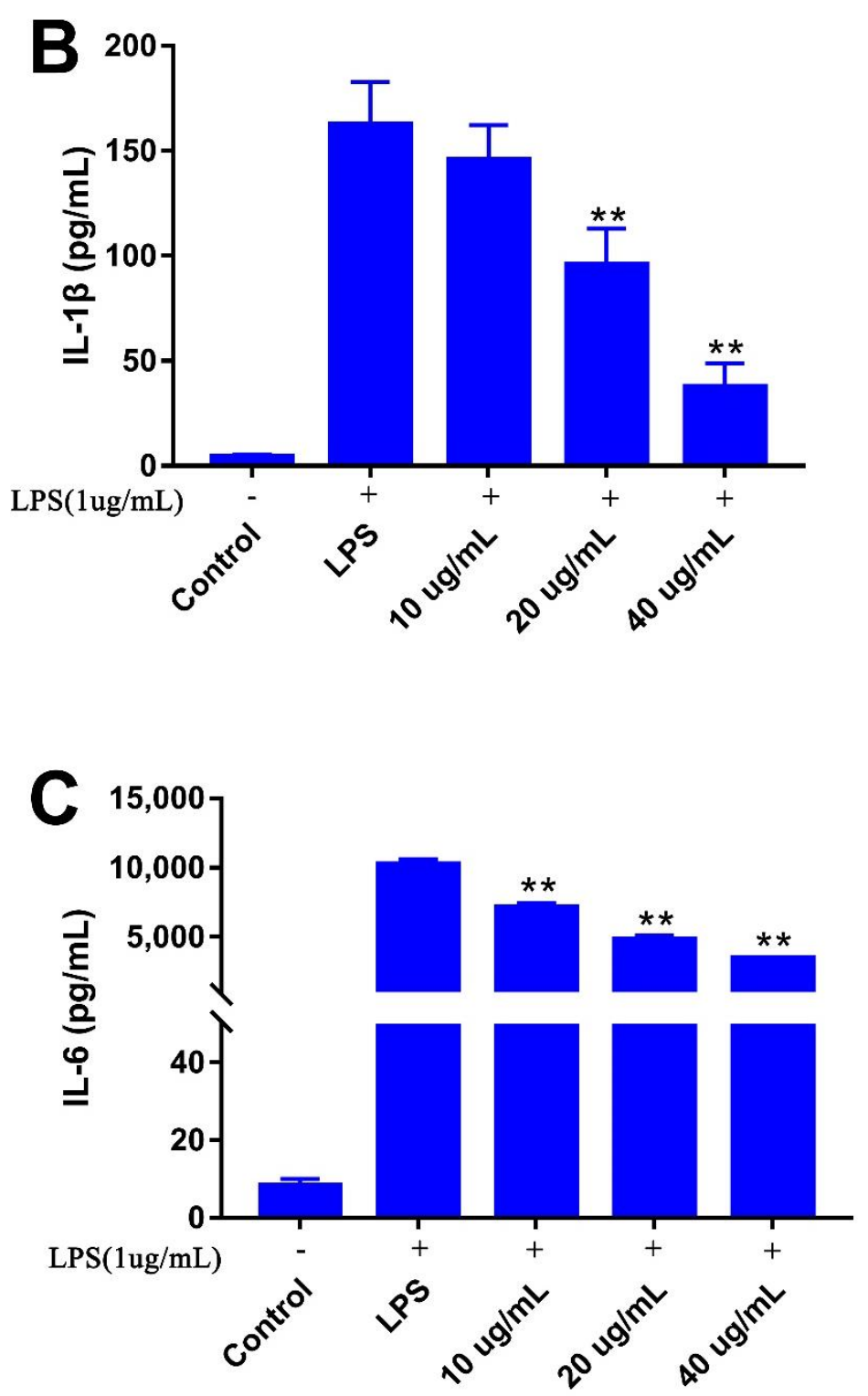

Figure 6. The effect of various concentrations of poricoic acid B on the productions of TNF- $\alpha$ (A), IL-1 $\beta$ (B), and IL-6 (C) in lipopolysaccharide (LPS)-stimulated murine macrophage cells (RAW 264.7). The values are presented as the mean $\pm \mathrm{SD}(n=3) .{ }^{*} p<0.05,{ }^{* *} p<0.01 \mathrm{vs.} \mathrm{LPS} \mathrm{group.}$

\section{Conclusions}

In this study, four triterpenoids were isolated from Poriae Cutis: two 3,4-seco-lanostan7,9(11)-diene type triterpenes (poricoic acid B and poricoic acid A) and two lanosta-7,9(11)diene type triterpenes (dehydrotrametenolic acid and dehydroeburicoic acid). The cytotoxicity of the 3,4-seco-lanostan-7,9(11)-diene type triterpenes was lower than that of the lanosta-7,9(11)-diene type triterpenes. The 3,4-seco-lanostan-7,9(11)-diene type triterpenes were found to inhibit the NO generation in LPS-induced RAW264.7 cells. However, the lanosta-7,9(11)-diene type triterpenes had no effect on the production of NO. In addition, poricoic acid B significantly inhibited the secretion of TNF- $\alpha$, IL-1 $\beta$, and IL- 6 in LPS-induced RAW 264.7 cells. Our results indicated that 3,4-seco-lanostan-7,9(11)-diene type triterpenes exhibited more anti-inflammatory activities than lanosta-7,9(11)-diene type triterpenes, and provided experimental evidence that Poriae Cutis is a potential source of natural anti-inflammatory agents for use in pharmaceuticals and functional foods. 
Supplementary Materials: The following are available online at https://www.mdpi.com/article/10 $.3390 /$ foods10123155/s1. Figure S1: ${ }^{1} \mathrm{H}$ - and ${ }^{13} \mathrm{C}-\mathrm{NMR}$ spectra of compound 1, Figure S2: ${ }^{1} \mathrm{H}-$ and ${ }^{13} \mathrm{C}$-NMR spectra of compound 2, Figure S3 ${ }^{1} \mathrm{H}$ - and ${ }^{13} \mathrm{C}-\mathrm{NMR}$ spectra of compound 3, Figure S4: ${ }^{1} \mathrm{H}$ - and ${ }^{13} \mathrm{C}-\mathrm{NMR}$ spectra of compound 4.

Author Contributions: Conceptualization, Y.L. and W.H.; methodology, L.Z. and M.Y.; software, L.Z.; validation, L.Z. and W.H.; formal analysis, L.Z.; investigation, L.Z. and M.Y.; resources, W.H.; data curation, L.Z.; writing—original draft preparation, L.Z. and M.Y.; writing—review and editing, X.F. and S.A.I.; visualization, L.Z.; supervision, L.Z.; project administration, W.H.; funding acquisition, Y.L. All authors have read and agreed to the published version of the manuscript.

Funding: This research was funded by the National Key Research and Development Plan of China (2017YFC1703006), and the Agricultural Science and Technology Innovation Center of Hubei Province (2016-620-000-001-044) supported by Hubei Agriculture Research System (HBHZDZB-2021-023).

Data Availability Statement: Not applicable.

Conflicts of Interest: The authors declare no conflict of interest.

\section{References}

1. Wang, W.H.; Dong, H.J.; Yan, R.Y.; Li, H.; Li, P.Y.; Chen, P.; Yang, B.; Wang, Z.M. Comparative study of lanostane-type triterpene acids in different parts of Poria cocos (Schw.) Wolf by UHPLC-Fourier transform MS and UHPLC-triple quadruple MS. J. Pharm. Biomed. Anal. 2015, 102, 203-214. [CrossRef]

2. Zhang, G.; Wang, H.; Xie, W.; Wang, Q.; Wang, X.; Wang, C.; Du, Y.; Huo, C.; Wang, Q. Comparison of triterpene compounds of four botanical parts from Poria cocos (Schw.) wolf using simultaneous qualitative and quantitative method and metabolomics approach. Food Res. Int. 2019, 121, 666-677. [CrossRef] [PubMed]

3. Feng, Y.L.; Lei, P.; Tian, T.; Yin, L.; Chen, D.Q.; Chen, H.; Mei, Q.B.; Zhao, Y.Y.; Lin, R.C. Diuretic activity of some fractions of the epidermis of Poria cocos. J. Ethnopharmacol. 2013, 150, 1114-1118. [CrossRef] [PubMed]

4. Wang, Y.-Z.; Zhang, J.; Zhao, Y.-L.; Li, T.; Shen, T.; Li, J.-Q.; Li, W.-Y.; Liu, H.-G. Mycology, cultivation, traditional uses, phytochemistry and pharmacology of Wolfiporia cocos (Schwein.) Ryvarden et Gilb.: A review. J. Ethnopharmacol. 2013, 147, 265-276. [CrossRef] [PubMed]

5. Zhu, L.X.; Xu, J.; Zhang, S.J.; Wang, R.J.; Huang, Q.; Chen, H.B.; Dong, X.P.; Zhao, Z.Z. Qualitatively and quantitatively comparing secondary metabolites in three medicinal parts derived from Poria cocos (Schw.) Wolf using UHPLC-QTOF-MS/MS-based chemical profiling. J. Pharm. Biomed. Anal. 2018, 150, 278-286. [CrossRef] [PubMed]

6. $\quad$ Li, N.; Yang, Y.-g.; Chen, Y.; Xu, R.; Gu, L.-h.; Xie, Y.-b.; Li, S.-m.; Zhan, C.-s.; Wang, Z.-t.; Yang, L. Analysis of triterpenoic acids in different medicinal parts of Poria cocos (Schw.) Wolf using supercritical fluid chromatography. Yaoxue Xuebao 2021, 56, 1120-1126.

7. Fu, M.; Wang, L.; Wang, X.; Deng, B.; Hu, X.; Zou, J. Determination of the Five Main Terpenoids in Different Tissues of Wolfiporia cocos. Molecules 2018, 23, 1839. [CrossRef] [PubMed]

8. Lee, S.R.; Lee, S.; Moon, E.; Park, H.-J.; Park, H.B.; Kim, K.H. Bioactivity-guided isolation of anti-inflammatory triterpenoids from the sclerotia of Poria cocos using LPS-stimulated Raw264.7 cells. Bioorganic Chem. 2017, 70, 94-99. [CrossRef]

9. Lee, S.; Lee, D.; Lee, S.O.; Ryu, J.-Y.; Choi, S.-Z.; Kang, K.S.; Kim, K.H. Anti-inflammatory activity of the sclerotia of edible fungus, Poria cocos Wolf and their active lanostane triterpenoids. J. Funct. Foods 2017, 32, 27-36. [CrossRef]

10. Cai, T.-G.; Cai, Y. Triterpenes from the Fungus Poria cocos and Their Inhibitory Activity on Nitric Oxide Production in Mouse Macrophages via Blockade of Activating Protein-1 Pathway. Chem. Biodivers. 2011, 8, 2135-2143. [CrossRef]

11. Giner, E.M.; Manez, S.; Recio, M.C.; Giner, R.M.; Cerda-Nicolas, M.; Rios, J.L. In vivo studies on the anti-inflammatory activity of pachymic and dehydrotumulosic acids. Planta Med. 2000, 66, 221-227. [CrossRef]

12. Akihisa, T.; Uchiyama, E.; Kikuchi, T.; Tokuda, H.; Suzuki, T.; Kimura, Y. Anti-Tumor-Promoting Effects of 25-Methoxyporicoic Acid A and Other Triterpene Acids from Poria cocos. J. Nat. Prod. 2009, 72, 1786-1792. [CrossRef]

13. Kikuchi, T.; Uchiyama, E.; Ukiya, M.; Tabata, K.; Kimura, Y.; Suzuki, T.; Akihisa, T. Cytotoxic and Apoptosis-Inducing Activities of Triterpene Acids from Poria cocos. J. Nat. Prod. 2011, 74, 137-144. [CrossRef] [PubMed]

14. Ukiya, M.; Akihisa, T.; Tokuda, H.; Hirano, M.; Oshikubo, M.; Nobukuni, Y.; Kimura, Y.; Tai, T.; Kondo, S.; Nishino, H. Inhibition of tumor-promoting effects by poricoic acids $\mathrm{G}$ and $\mathrm{H}$ and other lanostane-type triterpenes and cytotoxic activity of poricoic acids A and G from Poria cocos. J. Nat. Prod. 2002, 65, 462-465. [CrossRef] [PubMed]

15. Zhao, Y.-Y.; Feng, Y.-L.; Du, X.; Xi, Z.-H.; Cheng, X.-L.; Wei, F. Diuretic activity of the ethanol and aqueous extracts of the surface layer of Poria cocos in rat. J. Ethnopharmacol. 2012, 144, 775-778. [CrossRef]

16. Mengzhou, Y.; Ying, L.; Lijia, Z.; Yi, W.; Wen, H. Isolation and preparation of poricoic acid A and poricoic acid B from poriae cutis by high-speed counter current chromatography. Food Sci. China 2020, 41, 179-184.

17. Liu, F.; Liu, Y.; Feng, X.; Ibrahim, S.A.; Huang, W. Structure characterization and in vitro immunomodulatory activities of carboxymethyl pachymaran. Int. J. Biol. Macromol. 2021, 178, 94-103. [CrossRef]

18. Sun, H.; Zhang, J.; Chen, F.; Chen, X.; Zhou, Z.; Wang, H. Activation of RAW264.7 macrophages by the polysaccharide from the roots of Actinidia eriantha and its molecular mechanisms. Carbohydr. Polym. 2015, 121, 388-402. [CrossRef] 
19. Zeng, H.L.; Liu, Q.; Yu, J.G.; Jiang, X.Y.; Wu, Z.L.; Wang, M.L.; Chen, M.; Chen, X.Q. One-step separation of nine structural analogues from Poria cocos (Schw.) Wolf. via tandem high-speed counter-current chromatography. J. Chromatogr. B Anal. Technol. Biomed. Life Sci. 2015, 1004, 10-16. [CrossRef]

20. Wang, Y.; Guo, L.; Liu, C.; Zhang, Y.; Li, S. Total Triterpenoid Extraction from Inonotus Obliquus Using Ionic Liquids and Separation of Potential Lactate Dehydrogenase Inhibitors via Ultrafiltration High-Speed Countercurrent Chromatography. Molecules 2021, 26, 2467. [CrossRef]

21. Yang, P.F.; Hua, T.; Wang, D.; Zhao, Z.W.; Xi, G.L.; Chen, Z.F. Phytochemical and chemotaxonomic study of Poria cocos (Schw.) Wolf. Biochem. Syst. Ecol. 2019, 83, 54-56. [CrossRef]

22. Dong, H.J.; Yan, R.Y.; Li, H.; Wang, W.H.; Wang, X.; Yang, B. Acid-Alkali Extraction of Triterpene Acids from Poria and Preparative Separation by High-Speed Counter-Current Chromatography. Sep. Sci. Technol. 2014, 49, 2765-2771. [CrossRef]

23. Tai, T.; Akahori, A.; Shingu, T. Triterpenoids from poria-cocos. Phytochemistry 1991, 30, 2796-2797. [CrossRef]

24. Yang, S.W.; Shen, Y.C.; Chen, C.H. Steroids and triterpenoids of Antrodia cinnamomea-A fungus parasitic on Cinnamomum micranthum. Phytochemistry 1996, 41, 1389-1392. [CrossRef]

25. Yongyi, Y.; Yajun, Z.; Cebin, H.; Xinyuan, Z.; Yang, L.; Yangyuan, X.; Lijun, Y. Chemistry and immunostimulatory activity of a polysaccharide from Undaria pinnatifida. Food Chem. Toxicol. 2019, 128, 119-128.

26. Dong, H.J.; Xue, Z.Z.; Geng, Y.L.; Wang, X.; Yang, B. Lanostane triterpenes isolated from epidermis of Poria cocos. Phytochem. Lett. 2017, 22, 102-106. [CrossRef]

27. Gao, Y.; Fang, L.; Cai, R.; Zong, C.; Chen, X.; Lu, J.; Qi, Y. Shuang-Huang-Lian exerts anti-inflammatory and anti-oxidative activities in lipopolysaccharide-stimulated murine alveolar macrophages. Phytomedicine 2014, 21, 461-469. [CrossRef] [PubMed]

28. Johnson, T.A.; Sohn, J.; Inman, W.D.; Bjeldanes, L.F.; Rayburn, K. Lipophilic stinging nettle extracts possess potent antiinflammatory activity, are not cytotoxic and may be superior to traditional tinctures for treating inflammatory disorders. Phytomedicine 2013, 20, 143-147. [CrossRef]

29. da Cunha, A.L.; Aguiar, J.A.K.; Correa da Silva, F.S.; Michelacci, Y.M. Do chondroitin sulfates with different structures have different activities on chondrocytes and macrophages? Int. J. Biol. Macromol. 2017, 103, 1019-1031. [CrossRef]

30. Jeong, Y.H.; Oh, Y.-C.; Cho, W.-K.; Yim, N.-H.; Ma, J.Y. Hoveniae Semen Seu Fructus Ethanol Extract Exhibits Anti-Inflammatory Activity via MAPK, AP-1, and STAT Signaling Pathways in LPS-Stimulated RAW 264.7 and Mouse Peritoneal Macrophages. Mediat. Inflamm. 2019, 2019, 9184769. [CrossRef] [PubMed]

31. Fang, Z.-J.; Zhang, T.; Chen, S.-X.; Wang, Y.-L.; Zhou, C.-X.; Mo, J.-X.; Wu, Y.-J.; Xu, Y.-K.; Lin, L.-G.; Gan, L.-S. Cycloartane triterpenoids from Actaea vaginata with anti-inflammatory effects in LPS-stimulated RAW264.7 macrophages. Phytochemistry 2019, 160, 1-10. [CrossRef] [PubMed]

32. Liu, W.; Deng, S.; Zhou, D.; Huang, Y.; Li, C.; Hao, L.; Zhang, G.; Su, S.; Xu, X.; Yang, R.; et al. 3,4-seco-Dammarane Triterpenoid Saponins with Anti-Inflammatory Activity Isolated from the Leaves of Cyclocarya paliurus. J. Agric. Food Chem. 2020, 68, 2041-2053. [CrossRef] [PubMed] 